\title{
Experimental Investigation of Phase Equilibria in the Fe-Si-Zr Ternary System
}

\author{
C.P. Wang, Y. Hu, S.Y. Yang, Y. Lu, Q.W. Jiang, and X.J. Liu
}

(Submitted January 15, 2013; in revised form April 8, 2013; published online May 8, 2013)

\begin{abstract}
The phase equilibria of the Fe-Si-Zr ternary system at 1273 and $1373 \mathrm{~K}$ have been experimentally investigated using scanning electron microscopy, electron probe microanalyzer and $x$-ray diffraction. In the present study, the established isothermal sections are characterized by a series of binary phases, ternary compounds and solid solutions. $\mathrm{FeZr}_{2}$ and $\mathrm{SiZr}_{2}$ do not form a continuous solid solution at 1273 and $1373 \mathrm{~K}$. A new phase denoted here as $\tau_{9}(29 \mathrm{Fe} 42 \mathrm{Si29Zr})$ was found at both temperatures. The measured composition of the $\tau_{8}$ phase agrees with the formula $\mathrm{Fe}_{4} \mathrm{Si}_{2} \mathrm{Zr}$ found in the previous structural investigations. The newly determined phase equilibria of the Fe-Si-Zr system will provide important information for the development of silicon steels.
\end{abstract}

Keywords electron microprobe, microstructure, phase diagrams, ternary alloy systems

\section{Introduction}

As a significant material utilized in electrical devices, such as transformers, the silicon steels play an important role on the technical features, operating costs and comprehensive performance of these devices. ${ }^{[1-4]}$ The magnetic flux $\left(\mathrm{B}_{10}\right)$ and core loss value $\left(\mathrm{W}_{17 / 50}\right)$ are basic parameters indicating the quality of the silicon steels, and both of them are sensitive to the composition of silicon steels. ${ }^{[5]}$ Additionally, it has been reported that $\mathrm{Zr}$ addition (0.05-0.3 wt.\%) into silicon steels can form a stable compound $\mathrm{ZrN}$, which will lead to the grain refinement, a decrease of iron loss and an obvious increase in the recrystallization temperature of the alloys. ${ }^{[6]}$ It can thus be seen that $\mathrm{Zr}$ element in the Fe-Si alloys has a great impact on the performance of silicon steels. Therefore, the Fe-Si-Zr ternary system is an important subsystem for the silicon steels.

The three binary systems Fe-Si, $\mathrm{Zr}-\mathrm{Fe}$ and $\mathrm{Si}-\mathrm{Zr}$, constituting the Fe-Si-Zr ternary system, are shown in Fig. 1. The Fe-Si binary phase diagram reviewed by Kubaschewski ${ }^{[7]}$ shows the presence of seven intermediate phases: $\xi_{\alpha}\left(\mathrm{FeSi}_{2}\right), \xi_{\beta}\left(\mathrm{FeSi}_{2}\right), \quad \varepsilon \quad(\mathrm{FeSi}), \quad \eta \quad\left(\mathrm{Fe}_{5} \mathrm{Si}_{3}\right)$, $\alpha_{1}\left(\mathrm{Fe}_{3} \mathrm{Si}\right), \alpha_{2}(\mathrm{Fe}-\mathrm{Si})$, and $\beta\left(\mathrm{Fe}_{2} \mathrm{Si}\right)$. The $\mathrm{Zr}-\mathrm{Fe}$ binary phase diagram was determined by Stein et al. ${ }^{[8]}$ Four intermediate phases exist: $\alpha\left(\mathrm{Fe}_{2} \mathrm{Zr}\right), \beta\left(\mathrm{Fe}_{2} \mathrm{Zr}\right), \mathrm{FeZr}_{3}$ and $\mathrm{FeZr}_{2}$. The Si-Zr binary phase diagram provided by Okamoto $^{[9]}$ depicts the following intermediate phases: $\mathrm{SiZr}_{3}, \mathrm{SiZr}_{2}, \mathrm{Si}_{3} \mathrm{Zr}_{5}, \mathrm{Si}_{2} \mathrm{Zr}_{3}, \alpha\left(\mathrm{Si}_{4} \mathrm{Zr}_{5}\right), \beta\left(\mathrm{Si}_{4} \mathrm{Zr}_{5}\right), \alpha(\mathrm{SiZr})$,

C.P. Wang, Y. Hu, S.Y. Yang, Y. Lu, and X.J. Liu, Department of Materials Science and Engineering, College of Materials, Xiamen University, Xiamen 361005, People's Republic of China; Q.W. Jiang, Anshan Iron and Steel Group Corporation, Anshan 114021, People's Republic of China. Contact e-mail: 1xj@xmu.edu.cn. $\beta$ ( $\mathrm{SiZr}$ ), and $\mathrm{Si}_{2} \mathrm{Zr}$. The stable solid phases and their crystal structures in the three binary systems are listed in Table 1.

Regarding the experimental data of phase equilibria in the Fe-Si-Zr system, only one partial isothermal section at $1073 \mathrm{~K}$ studied by Lisenko et al. ${ }^{[10]}$ is available, as shown in Fig. 1. The data and ternary compounds in this system were reviewed by Raghavan ${ }^{[11]}$ and $\mathrm{Du}$ et al. ${ }^{[12]}$ Furthermore, the existence of ternary compounds is reported: $\tau_{1}$ $(\mathrm{FeSiZr}),{ }^{[10,13]} \tau_{2}\left(\mathrm{Fe}_{3} \mathrm{SiZr}_{2}\right),{ }^{[10,14]} \tau_{3}\left(\mathrm{Fe}_{4} \mathrm{Si}_{4} \mathrm{Zr}_{7}\right),{ }^{[10,13,15]} \tau_{4}$ $\left(\mathrm{Fe}_{2} \mathrm{Si}_{2} \mathrm{Zr}\right),{ }^{[10,16]} \tau_{5}\left(\mathrm{Fe}_{16} \mathrm{Si}_{7} \mathrm{Zr}_{6}\right),{ }^{[10,17]} \tau_{6}\left(\mathrm{Fe}_{5} \mathrm{Si}_{12} \mathrm{Zr}_{3}\right){ }^{[18]} \tau_{7}$ $\left(\mathrm{Fe}_{2} \mathrm{Si}_{3} \mathrm{Zr}_{3}\right),{ }^{[19]} \tau_{8}\left(\mathrm{Fe}_{4} \mathrm{Si}_{2} \mathrm{Zr}\right),{ }^{[20,21]}(\mathrm{Fe}, \mathrm{Zr})_{5} \mathrm{Si}_{3},{ }^{[10,18]}(\mathrm{Fe}, \mathrm{Si})_{2}$ $\mathrm{Zr}$, ${ }^{[10,18,22]}$ see Table 1. The structures of the $\tau_{4}\left(\mathrm{Fe}_{2} \mathrm{Si}_{2} \mathrm{Zr}\right)$, $\tau_{5}\left(\mathrm{Fe}_{16} \mathrm{Si}_{7} \mathrm{Zr}_{6}\right), \tau_{6}\left(\mathrm{Fe}_{5} \mathrm{Si}_{12} \mathrm{Zr}_{3}\right), \tau_{7}\left(\mathrm{Fe}_{2} \mathrm{Si}_{3} \mathrm{Zr}_{3}\right), \tau_{8}\left(\mathrm{Fe}_{4} \mathrm{Si}_{2} \mathrm{Zr}\right)$, $(\mathrm{Fe}, \mathrm{Zr})_{5} \mathrm{Si}_{3}$ and $(\mathrm{Fe}, \mathrm{Si})_{2} \mathrm{Zr}$ have not been assigned PDF numbers (calculated powder diffraction data), except $\tau_{1}$ (FeSiZr), $\tau_{2}\left(\mathrm{Fe}_{3} \mathrm{SiZr}_{2}\right)$ and $\tau_{3}\left(\mathrm{Fe}_{4} \mathrm{Si}_{4} \mathrm{Zr}_{7}\right)$. Regarding the isothermal section of the $\mathrm{Fe}-\mathrm{Si}-\mathrm{Zr}$ system at $1073 \mathrm{~K}$, the addition of about 10-20 at.\% $\mathrm{Zr}$ stabilizes a ternary phase of the $\mathrm{Fe}_{5} \mathrm{Si}_{3}$ structure at 37.5 at.\% $\mathrm{Si}^{[10]}$ Here, it should be noted that limited experimental data will restrict the compositional design of silicon steels. Only one partial isothermal section at $1073 \mathrm{~K}$ is not enough to do research for compositional design of silicon steels, while considering that the heat treatment temperature of silicon steels belongs to high-temperature range. Therefore, it is necessary to comprehensively determine the phase equilibria of the Fe-Si-Zr ternary system, especially at high temperature. The present research has thus been done to tackle this issue. In this study, the phase equilibria of the Fe-Si-Zr ternary system at 1273 and $1373 \mathrm{~K}$ were experimentally investigated by using scanning electron microscopy (back-scattered electron, $\mathrm{BSE}$ ), electron probe microanalyzer (EPMA) and x-ray diffraction (XRD).

\section{Experimental Procedure}

Iron (99.8 wt.\%), zirconium (99.8 wt.\%) and silicon (99.9 wt.\%) were used as starting materials. Bulk alloy 


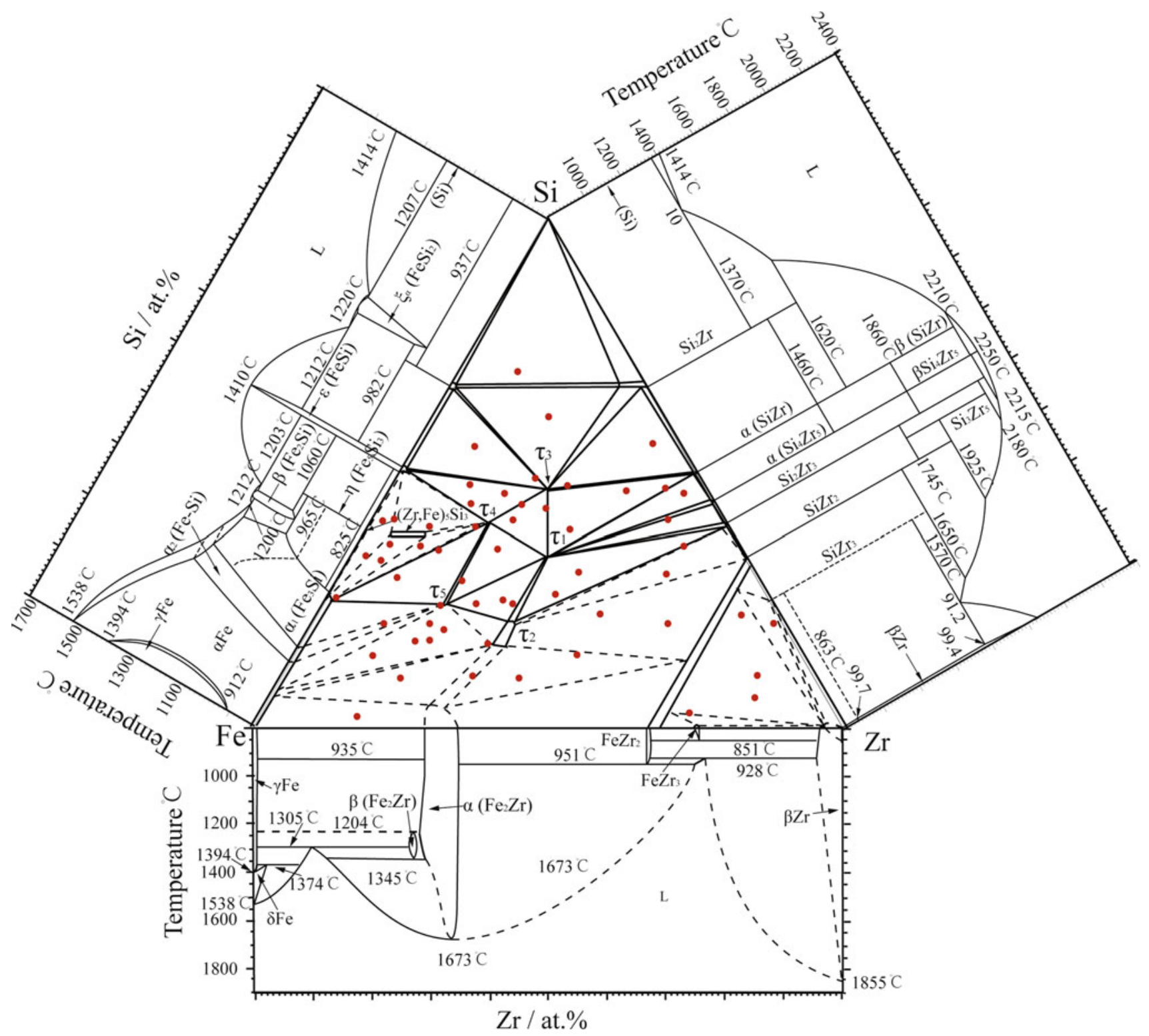

Fig. 1 Binary phase diagrams bonding the Fe-Si-Zr ternary system ${ }^{[7-9]}$ and experimentally determined isothermal sections of the Fe-Si$\mathrm{Zr}$ system at $1073 \mathrm{~K}^{[10]}$

buttons were prepared from pure elements by arc melting under a high purity argon atmosphere using a non-consumable tungsten electrode. The ingots were melted at least 5 times in order to achieve homogeneity. The sample weight was around $20 \mathrm{~g}$ and the overall weight loss during melting was generally less than $0.30 \mathrm{wt} . \%$. Afterwards, the ingots were cut into small pieces by wire-cutting machine for heat treatment and further observations.

All samples were sealed in quartz capsules evacuated and backfilled with argon gas. The specimens were annealed at $1273 \mathrm{~K}$ for $1440 \mathrm{~h}$ and $1373 \mathrm{~K}$ for $720 \mathrm{~h}$, respectively. After the heat treatment, the specimens were quenched into ice water.

After annealing and standard metallographic preparation, the equilibrium compositions of the phases were measured by EPMA (JXA-8100R, JEOL, Japan) with the average value of seven measurements. Pure elements were used as standards and the measurements were carried out at $20.0 \mathrm{kV}$ for voltage and $10 \mathrm{~mA}$ for beam current, respectively. XRD was used to identify the crystal structure of the constituent phases. The XRD measurement was carried out on a Phillips Panalytical X-pert diffractometer using $\mathrm{CuK}_{\alpha}$ radiation at $40.0 \mathrm{kV}$ and $30 \mathrm{~mA}$. The data were collected in the range of $2 \theta$ from $20^{\circ}$ to $90^{\circ}$ at a step of $0.0167^{\circ}$.

\section{Results and Discussion}

\subsection{Microstructure and Phase Equilibria}

BSE images of typical ternary Fe-Si-Zr alloys annealed at $1273 \mathrm{~K}$ and $1373 \mathrm{~K}$ are presented in Fig. 2 to 4 . Phase 
Table 1 The stable solid phases in the three binary systems

\begin{tabular}{|c|c|c|c|c|c|c|c|}
\hline System & Phase & Pearson's symbol & Prototype & Space Group & Strukturbericht & Lattice parameters, pm & References \\
\hline \multirow[t]{9}{*}{$\mathrm{Fe}-\mathrm{Si}$} & $\xi_{\alpha}\left(\mathrm{FeSi}_{2}\right)$ & $t P 3$ & $\mathrm{FeSi}_{2}$ & $P 4 / m m m$ & $\ldots$ & $a=286.65$ & {$[7]$} \\
\hline & $\varepsilon(\mathrm{FeSi})$ & $c P 8$ & $\mathrm{FeSi}$ & $P 2_{1} 3$ & $B 20$ & $a=246.8, c=396.0$ & [7] \\
\hline & $\eta\left(\mathrm{Fe}_{5} \mathrm{Si}_{3}\right)$ & $h P 16$ & $\mathrm{Mn}_{5} \mathrm{Si}_{3}$ & $\mathrm{P}_{3} / \mathrm{mcm}$ & $D 8_{8}$ & $a=675.9, c=472.0$ & [7] \\
\hline & $\beta\left(\mathrm{Fe}_{2} \mathrm{Si}\right)$ & $h P 6$ & $\mathrm{Fe}_{2} \mathrm{Si}$ & $P \overline{3} \mathrm{~m} 1$ & $\ldots$ & $a=405.2, c=508.55$ & {$[7]$} \\
\hline & $\alpha_{1}\left(\mathrm{Fe}_{3} \mathrm{Si}\right)$ & $c F 16$ & $\mathrm{BiF}_{3}$ & $F m \overline{3} m$ & $D 0_{3}$ & $a=565$ & {$[7]$} \\
\hline & $\alpha_{2}(\mathrm{Fe}-\mathrm{Si})$ & $c P 2$ & $\mathrm{CsCl}$ & $P m \overline{3} m$ & $B 2$ & $a=281$ & {$[7]$} \\
\hline & $\alpha \mathrm{Fe}$ & $c 12$ & $\mathrm{~W}$ & $\operatorname{Im} \overline{3} m$ & $A 2$ & $a=286.65$ & {$[7]$} \\
\hline & $\xi_{\beta}\left(\mathrm{FeSi}_{2}\right)$ & $o C 48$ & $\mathrm{FeSi}_{2}$ & Cmca & $\ldots$ & $a=986.3, b=779.1, c=783.3$ & {$[7]$} \\
\hline & $\gamma \mathrm{Fe}$ & $c F 4$ & $\mathrm{Cu}$ & $F m \overline{3} m$ & $A 1$ & $a=366.60$ & [7] \\
\hline \multirow[t]{4}{*}{$\mathrm{Zr}-\mathrm{Fe}$} & $\alpha\left(\mathrm{ZrFe}_{2}\right)$ & $c F 24$ & $\mathrm{MgCu}_{2}$ & $F d \overline{3} m$ & $C 15$ & $a=0.702$ & {$[8]$} \\
\hline & $\beta\left(\mathrm{ZrFe}_{2}\right)$ & $h P 24$ & $\mathrm{MgNi}_{2}$ & $P 6_{3} / m m c$ & $C 36$ & $a=0.495, c=1.614$ & {$[8]$} \\
\hline & $\mathrm{Zr}_{2} \mathrm{Fe}$ & $t I 12$ & $\mathrm{CuAl}_{2}$ & $I 4 / \mathrm{mcm}$ & $C 16$ & $a=0.638, c=0.560$ & {$[8]$} \\
\hline & $\mathrm{Zr}_{3} \mathrm{Fe}$ & $o C 16$ & $\mathrm{Re}_{3} \mathrm{~B}$ & $\mathrm{Cmcm}$ & $E 1_{a}$ & $a=0.332, b=1.100, c=0.882$ & {$[8]$} \\
\hline \multirow[t]{12}{*}{$\mathrm{Si}-\mathrm{Zr}$} & $\mathrm{SiZr}_{3}$ & $t P 32$ & $\mathrm{Ti}_{3} \mathrm{P}$ & $P 4_{2} / n$ & $\ldots$ & $a=1101, c=545$ & [9] \\
\hline & $\mathrm{SiZr}_{2}$ & $t I 12$ & $\mathrm{CuAl}_{2}$ & $\mathrm{I} / \mathrm{mcm}$ & $C 16$ & $a=660.9, c=529.8$ & [9] \\
\hline & $\mathrm{Si}_{2} \mathrm{Zr}_{3}$ & $t P 10$ & $\mathrm{Si}_{2} \mathrm{U}_{3}$ & $\mathrm{P} 4 / \mathrm{mbm}$ & $D 5 a$ & $a=708.2, c=371.5$ & [9] \\
\hline & $\alpha\left(\mathrm{Si}_{4} \mathrm{Zr}_{5}\right)$ & $t P 36$ & $\mathrm{Si}_{4} \mathrm{Zr}_{5}$ & $P 4_{1} 2_{1} 2$ & $\ldots$ & $a=712.25, c=1300.0$ & {$[9]$} \\
\hline & $\alpha(\mathrm{SiZr})$ & $o P 8$ & $\mathrm{FeB}$ & Pnma & $B 27$ & $a=699.5, b=378.6, c=529.6$ & [9] \\
\hline & $\mathrm{Si}_{2} \mathrm{Zr}$ & $o C 12$ & $\mathrm{Si}_{2} \mathrm{Zr}$ & $\mathrm{Cmcm}$ & $C 49$ & $a=372.1, b=1468, c=368.3$ & [9] \\
\hline & $\beta(\mathrm{SiZr})$ & $o C 8$ & $C r B$ & $\mathrm{Cmcm}$ & $\ldots$ & $\ldots$ & [9] \\
\hline & $\beta\left(\mathrm{Si}_{4} \mathrm{Zr}_{5}\right)$ & $\ldots$ & $\ldots$ & $\ldots$ & $\ldots$ & $\ldots$ & [9] \\
\hline & $\mathrm{Si}_{3} \mathrm{Zr}_{5}$ & $h P 16$ & $\mathrm{Mn}_{5} \mathrm{Si}_{3}$ & $\mathrm{P}_{3} / \mathrm{mcm}$ & $D 8_{8}$ & $a=788.6, c=555.8$ & [9] \\
\hline & $\mathrm{Si}$ & $c F 8$ & $\mathrm{C}$ & $F d \overline{3} m$ & $A 4$ & $a=543.06$ & [9] \\
\hline & $\alpha \mathrm{Zr}$ & $h P 2$ & $\mathrm{Mg}$ & $P 6_{3 / m m c}$ & $A 3$ & $a=323.2, c=514.7$ & [9] \\
\hline & $\beta \mathrm{Zr}$ & $c / 2$ & $\mathrm{~W}$ & $\operatorname{Im} \overline{3} m$ & $A 2$ & $a=356.8$ & [9] \\
\hline \multirow[t]{10}{*}{$\mathrm{Fe}-\mathrm{Si}-\mathrm{Zr}$} & $\tau_{1}(\mathrm{FeSiZr})$ & $o P 12$ & TiNiSi & Pnma & $C 23$ & $a=640.5, b=393.5, c=719.9$ & {$[10,13]$} \\
\hline & $\tau_{2}\left(\mathrm{Fe}_{3} \mathrm{SiZr}_{2}\right)$ & $h P 12$ & $\mathrm{MgZn}_{2}$ & $P 6_{3} / m m c$ & $\cdots$ & $a=498.5, c=809.6$ & {$[10,14]$} \\
\hline & $\tau_{3}\left(\mathrm{Fe}_{4} \mathrm{Si}_{7} \mathrm{Zr}_{4}\right)$ & $t I 60$ & $\mathrm{Zr}_{4} \mathrm{Co}_{4} \mathrm{Ge}_{7}$ & $I 4 / \mathrm{mmm}$ & $\ldots$ & $a=1305.6, c=509.0$ & {$[10,13,15]$} \\
\hline & $\tau_{4}\left(\mathrm{Fe}_{2} \mathrm{Si}_{2} \mathrm{Zr}\right)$ & $t P 10$ & $\mathrm{Al}_{2} \mathrm{Ga}_{2} \mathrm{Ge}$ & $I 4 / \mathrm{mmm}$ & $\mathrm{DI}_{3}$ & $a=375.0, c=966.0$ & {$[10,16]$} \\
\hline & $\tau_{5}\left(\mathrm{Fe}_{16} \mathrm{Si}_{7} \mathrm{Zr}_{6}\right)$ & $c F 116$ & $\mathrm{Mg}_{6} \mathrm{Cu}_{16} \mathrm{Si}_{7}$ & $F m \overline{3} m$ & $\cdots$ & $a=1168.0$ & {$[10,17]$} \\
\hline & $\tau_{6}\left(\mathrm{Fe}_{5} \mathrm{Si}_{12} \mathrm{Zr}_{3}\right)$ & $\ldots$ & $\ldots$ & $\ldots$ & $\ldots$ & $a=1430.0, b=1904.0, c=515.0$ & {$[18]$} \\
\hline & $\tau_{7}\left(\mathrm{Fe}_{2} \mathrm{Si}_{3} \mathrm{Zr}_{3}\right)$ & $o P 32$ & $\ldots$ & Pnma & $\ldots$ & $a=389.8, b=974.3, c=1304.2$ & [19] \\
\hline & $\tau_{8}\left(\mathrm{Fe}_{4} \mathrm{Si}_{2} \mathrm{Zr}\right)$ & $t P 14$ & $\mathrm{Fe}_{4} \mathrm{Si}_{2} \mathrm{Zr}$ & $P 4_{2} / \mathrm{mmm}$ & $\ldots$ & $a=375.0, c=966.0$ & {$[20,21]$} \\
\hline & $(\mathrm{Fe}, \mathrm{Zr})_{5} \mathrm{Si}_{3}$ & $h P 16$ & $\mathrm{Mn}_{5} \mathrm{Si}_{3}$ & $\mathrm{~Pb}_{3} / \mathrm{mcm}$ & $D 8_{8}$ & $a=674.0-677.0, c=472.0-474.0$ & {$[10,18]$} \\
\hline & $(\mathrm{Fe}, \mathrm{Si})_{2} \mathrm{Zr}$ & $h P 12$ & $\mathrm{MgZn}_{2}$ & $P 6_{3} / m m c$ & $C 14$ & $a=498.9, c=811.0$ & {$[10,18,22]$} \\
\hline
\end{tabular}

identification was based on the equilibrium composition measured by EPMA (Tables 2, 3) and XRD results (Fig. 5). Comparing with isothermal section at $1073 \mathrm{~K}$ shown in Fig. 1 , the ternary phases $\left(\tau_{1}, \tau_{2}, \tau_{3}, \tau_{4}, \tau_{5}\right)$, the phase $(\mathrm{Zr}, \mathrm{Fe})_{5} \mathrm{Si}_{3}$ and the large solubility of $\mathrm{Fe}$ in the $\mathrm{Si}_{2} \mathrm{Zr}$ also appear at $1273 \mathrm{~K}$ and $1373 \mathrm{~K}$. However, in this study, $\mathrm{FeZr}_{2}$ and $\mathrm{SiZr}_{2}$ do not form a continuous solid solution at 1273 and $1373 \mathrm{~K}$. The phase $\tau_{8}(57 \mathrm{Fe} 29 \mathrm{Si14Zr})$ appears at $1273 \mathrm{~K}$ and $1373 \mathrm{~K}$, which agrees with the formula $\mathrm{Fe}_{4} \mathrm{Si}_{2} \mathrm{Zr}$ found in the structural investigations of Yarmolyuk et al. ${ }^{[20,21]}$ and listed in the review of Raghavan. ${ }^{[11]}$ In addition, a new phase $\tau_{9}$ was observed in the present work, which indicates the existence of a new phase.

Figure 2(a) shows the three-phase microstructure $\left(\varepsilon(\mathrm{FeSi})+\xi_{\alpha}\left(\mathrm{FeSi}_{2}\right)+\tau_{3}\left(\mathrm{Fe}_{4} \mathrm{Si}_{7} \mathrm{Zr}_{4}\right)\right)$ of the $\mathrm{Fe}_{35} \mathrm{Si}_{55} \mathrm{Zr}_{10}$ (at.\%) alloy annealed at $1273 \mathrm{~K}$ for $1440 \mathrm{~h}$. A three-phase equilibrium $\left((\mathrm{Zr}, \mathrm{Fe})_{5} \mathrm{Si}_{3}+\tau_{8}\left(\mathrm{Fe}_{4} \mathrm{Si}_{2} \mathrm{Zr}\right)+\tau_{9}\right)$ was identified in the $\mathrm{Fe}_{50} \mathrm{Si}_{35} \mathrm{Zr}_{15}$ (at.\%) alloy annealed at $1273 \mathrm{~K}$ for $1440 \mathrm{~h}$, and indicated in Fig. 2(b). The homogeneity of phase $\tau_{9}$ ranges from 40.7 to 43.2 at. $\% \mathrm{Si}$, from 27.8 to 30.3 at.\% Zr, and from 27.2 to 28.8 at.\% Fe. Considering the composition of $\tau_{7}\left(\mathrm{Fe}_{2} \mathrm{Si}_{3} \mathrm{Zr}_{3}\right)$ given by Raghavan ${ }^{[11]}$ and the standard deviation of the measured concentration, $\tau_{9}$ may not be $\tau_{7}\left(\mathrm{Fe}_{2} \mathrm{Si}_{3} \mathrm{Zr}_{3}\right)$, ${ }^{[19]}$ which appears in the isothermal section at $1273 \mathrm{~K}^{[11]}$ Figure 2(c) presents the three-phase microstructure $\left((\mathrm{Zr}, \mathrm{Fe})_{5} \mathrm{Si}_{3}+\alpha \mathrm{Fe}+\tau_{8}\left(\mathrm{Fe}_{4} \mathrm{~S}-\right.\right.$ $\mathrm{i}_{2} \mathrm{Zr}$ )) of the $\mathrm{Fe}_{60} \mathrm{Si}_{30} \mathrm{Zr}_{10}$ (at.\%) alloy equilibrated at $1273 \mathrm{~K}$ for $1440 \mathrm{~h}$. In the $\mathrm{Fe}_{42} \mathrm{Si}_{39} \mathrm{Zr}_{19}$ (at.\%) alloy annealed at $1273 \mathrm{~K}$ for $1440 \mathrm{~h}$, a three-phase equilibrium $\left((\mathrm{Zr}, \mathrm{Fe})_{5} \mathrm{Si}_{3}+\tau_{9}+\tau_{4}\left(\mathrm{Fe}_{2} \mathrm{Si}_{2} \mathrm{Zr}\right)\right)$ was found, and shown in Fig. 2(d). Two three-phase equilibria of the $\left(\tau_{4}\left(\mathrm{Fe}_{2} \mathrm{~S}-\right.\right.$ $\left.\left.\mathrm{i}_{2} \mathrm{Zr}\right)+\tau_{9}+\varepsilon(\mathrm{FeSi})\right)$ and $\left(\tau_{5}+\tau_{8}\left(\mathrm{Fe}_{4} \mathrm{Si}_{2} \mathrm{Zr}\right)+\tau_{1}(\mathrm{Fe}-\right.$ SiZr)) were observed in the $\mathrm{Fe}_{41} \mathrm{Si}_{44} \mathrm{Zr}_{15}$ (at.\%) and $\mathrm{Fe}_{51.5} \mathrm{Si}_{27.5} \mathrm{Zr}_{21}$ (at.\%) alloys both quenched from $1273 \mathrm{~K}$ for 1440 h, and indicated in Fig. 2(e) and (f), respectively. Figure 3(a) shows the three-phase microstructure $\left(\alpha\left(\mathrm{Fe}_{2} \mathrm{Zr}\right)+\gamma \mathrm{Fe}+\tau_{5}\left(\mathrm{Fe}_{16} \mathrm{Si}_{7} \mathrm{Zr}_{6}\right)\right)$ of the $\mathrm{Fe}_{68} \mathrm{Si}_{12} \mathrm{Zr}_{20}$ (at.\%) alloy annealed at $1273 \mathrm{~K}$ for $1440 \mathrm{~h}$, where the gray, 

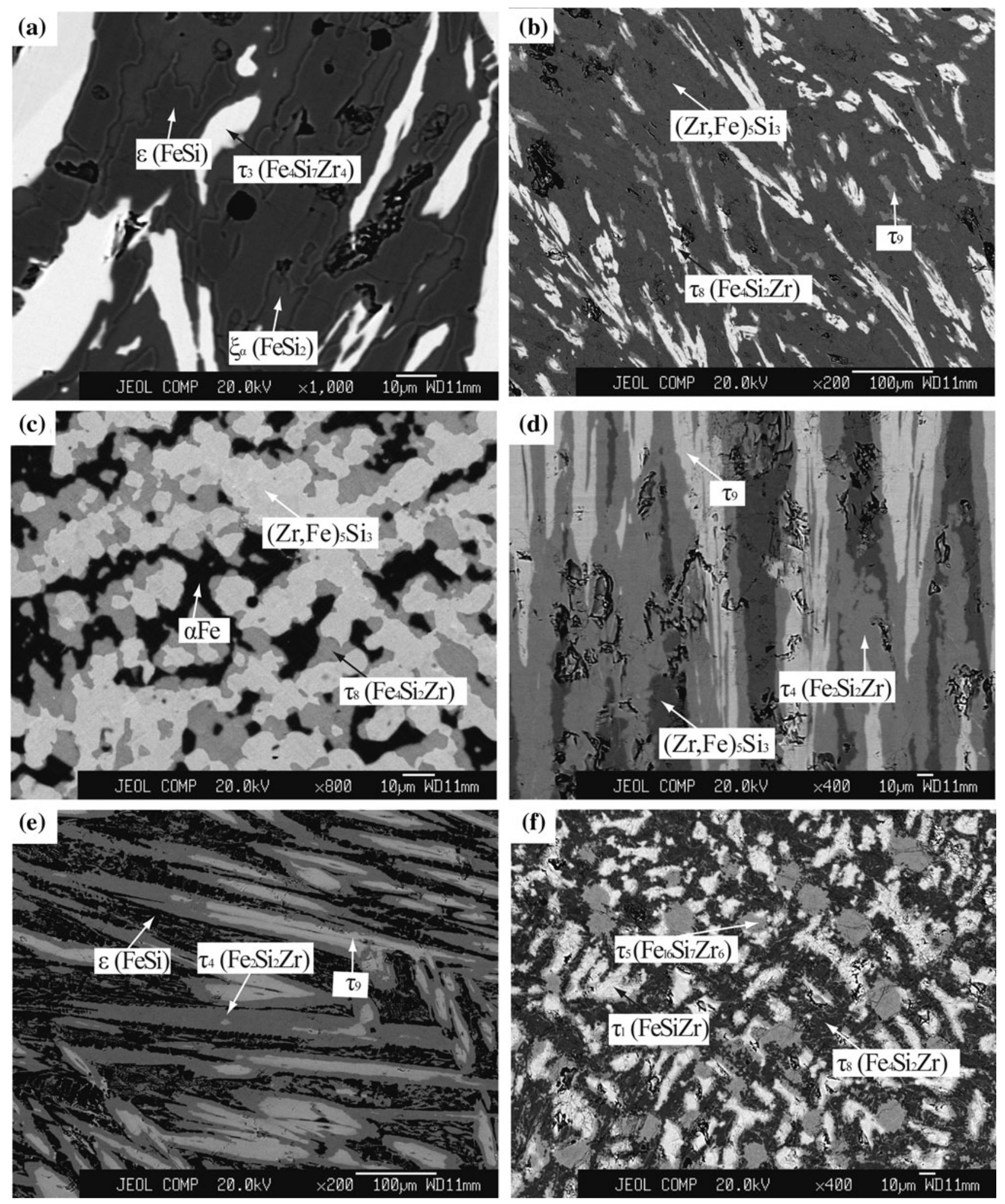

Fig. 2 BSE images of typical ternary alloys annealed at $1273 \mathrm{~K}$ for $1440 \mathrm{~h}$ : (a) the $\mathrm{Fe}_{35} \mathrm{Si}_{55} \mathrm{Zr}_{10}$ (at.\%); the $\mathrm{Fe}_{50} \mathrm{Si}_{35} \mathrm{Zr}_{15}$ (at.\%); (c) the $\mathrm{Fe}_{60} \mathrm{Si}_{30} \mathrm{Zr}_{10}$ (at.\%); (d) the $\mathrm{Fe}_{42} \mathrm{Si}_{39} \mathrm{Zr}_{19}$ (at.\%); (e) the $\mathrm{Fe}_{41} \mathrm{Si}_{44} \mathrm{Zr}_{15}$ (at.\%); and (f) the $\mathrm{Fe}_{51.5} \mathrm{Si}_{27.5} \mathrm{Zr}_{21}$ (at.\%) 
Table 2 Equilibrium compositions of the Fe-Si-Zr system determined at $1273 \mathrm{~K}$

\begin{tabular}{|c|c|c|c|c|c|c|c|}
\hline \multirow[b]{3}{*}{ Alloy, at.\% } & \multirow{3}{*}{$\begin{array}{c}\text { Phase equilibrium } \\
\text { Phase } 1+\text { Phase } 2+\text { Phase } 3\end{array}$} & \multicolumn{6}{|c|}{ Composition, at. $\%$} \\
\hline & & \multicolumn{2}{|c|}{ Phase 1} & \multicolumn{2}{|c|}{ Phase 2} & \multicolumn{2}{|c|}{ Phase 3} \\
\hline & & $\mathbf{S i}$ & $\mathbf{Z r}$ & $\mathbf{S i}$ & $\mathbf{Z r}$ & $\mathbf{S i}$ & $\mathbf{Z r}$ \\
\hline $\mathrm{Fe}_{20} \mathrm{Si}_{70} \mathrm{Zr}_{10}$ & $(\mathrm{Si})+\mathrm{Si}_{2} \mathrm{Zr}+\xi_{\alpha}\left(\mathrm{FeSi}_{2}\right)$ & 99.5 & 0.1 & 63.0 & 29.5 & 66.7 & 7.5 \\
\hline $\mathrm{Fe}_{35} \mathrm{Si}_{55} \mathrm{Zr}_{10}$ & $\varepsilon(\mathrm{FeSi})+\xi_{\alpha}\left(\mathrm{FeSi}_{2}\right)+\tau_{3}$ & 51.5 & 0.1 & 66.6 & 7.1 & 51.8 & 23.4 \\
\hline $\mathrm{Fe}_{20} \mathrm{Si}_{60} \mathrm{Zr}_{20}$ & $\xi_{\alpha}\left(\mathrm{FeSi}_{2}\right)+\mathrm{Si}_{2} \mathrm{Zr}+\tau_{3}$ & 66.5 & 6.9 & 63.9 & 27.9 & 53.0 & 22.8 \\
\hline $\mathrm{Fe}_{5} \mathrm{Si}_{55} \mathrm{Zr}_{40}$ & $\mathrm{Si}_{2} \mathrm{Zr}+\alpha(\mathrm{SiZr})+\tau_{3}$ & 62.6 & 30.3 & 50.6 & 48.8 & 50.3 & 25.7 \\
\hline $\mathrm{Fe}_{27.5} \mathrm{Si}_{49} \mathrm{Zr}_{23.5}$ & $\varepsilon(\mathrm{FeSi})+\tau_{3}+\tau_{9}$ & 50.4 & 0.3 & 50.5 & 24.3 & 43.2 & 28.3 \\
\hline $\mathrm{Fe}_{17} \mathrm{Si}_{48} \mathrm{Zr}_{35}$ & $\tau_{3}+\alpha(\operatorname{SiZr})+\tau_{9}$ & 49.0 & 25.6 & 50.1 & 49.7 & 42.9 & 28.7 \\
\hline $\mathrm{Fe}_{41} \mathrm{Si}_{44} \mathrm{Zr}_{15}$ & $\varepsilon(\mathrm{FeSi})+\tau_{4}+\tau_{9}$ & 49.8 & 0.1 & 40.5 & 19.5 & 42.8 & 27.8 \\
\hline $\mathrm{Fe}_{7} \mathrm{Si}_{46} \mathrm{Zr}_{47}$ & $\alpha(\mathrm{SiZr})+\alpha\left(\mathrm{Si}_{4} \mathrm{Zr}_{5}\right)+\tau_{9}$ & 50.1 & 49.1 & 45.5 & 54.2 & 42.5 & 30.3 \\
\hline $\mathrm{Fe}_{50} \mathrm{Si}_{40} \mathrm{Zr}_{10}$ & $\varepsilon(\mathrm{FeSi})+(\mathrm{Zr}, \mathrm{Fe})_{5} \mathrm{Si}_{3}+\tau_{4}$ & 49.9 & 0.0 & 36.0 & 10.4 & 41.3 & 17.5 \\
\hline $\mathrm{Fe}_{42} \mathrm{Si}_{39} \mathrm{Zr}_{19}$ & $\tau_{4}+(\mathrm{Zr}, \mathrm{Fe})_{5} \mathrm{Si}_{3}+\tau_{9}$ & 40.7 & 19.2 & 36.2 & 11.4 & 41.9 & 29.3 \\
\hline $\mathrm{Fe}_{50} \mathrm{Si}_{35} \mathrm{Zr}_{15}$ & $\tau_{9}+(\mathrm{Zr}, \mathrm{Fe})_{5} \mathrm{Si}_{3}+\tau_{8}$ & 40.6 & 29.3 & 35.1 & 11.5 & 29.3 & 13.9 \\
\hline $\mathrm{Fe}_{40} \mathrm{Si}_{35} \mathrm{Zr}_{25}$ & $\tau_{1}+\tau_{9}+\tau_{8}$ & 35.0 & 30.5 & 41.3 & 27.9 & 29.4 & 13.1 \\
\hline $\mathrm{Fe}_{60} \mathrm{Si}_{30} \mathrm{Zr}_{10}$ & $\alpha \mathrm{Fe}+(\mathrm{Zr}, \mathrm{Fe})_{5} \mathrm{Si}_{3}+\tau_{8}$ & 27.2 & 0.1 & 36.4 & 11.3 & 29.8 & 13.8 \\
\hline $\mathrm{Fe}_{51.5} \mathrm{Si}_{27.5} \mathrm{Zr}_{21}$ & $\tau_{1}+\tau_{5}+\tau_{8}$ & 33.9 & 32.2 & 24.4 & 20.0 & 28.8 & 14.2 \\
\hline $\mathrm{Fe}_{68} \mathrm{Si}_{19} \mathrm{Zr}_{13}$ & $\alpha \mathrm{Fe}+\tau_{5}+\tau_{8}$ & 11.8 & 0.6 & 24.0 & 19.9 & 28.5 & 14.0 \\
\hline $\mathrm{Fe}_{62.5} \mathrm{Si}_{16.5} \mathrm{Zr}_{21}$ & $\tau_{1}+\tau_{2}+\mathrm{Si}_{2} \mathrm{Zr}_{3}$ & 34.1 & 32.2 & 17.7 & 35.1 & 39.0 & 60.6 \\
\hline $\mathrm{Fe}_{68} \mathrm{Si}_{12} \mathrm{Zr}_{20}$ & $\gamma \mathrm{Fe}+\alpha\left(\mathrm{Fe}_{2} \mathrm{Zr}\right)+\tau_{5}$ & 2.8 & 0.3 & 14.0 & 18.3 & 12.4 & 23.2 \\
\hline $\mathrm{Fe}_{20} \mathrm{Si}_{20} \mathrm{Zr}_{60}$ & Liquid $+\mathrm{SiZr}_{2}+\alpha\left(\mathrm{Fe}_{2} \mathrm{Zr}\right)$ & 0.5 & 67.0 & 31.4 & 66.1 & 3.0 & 34.1 \\
\hline $\mathrm{Fe}_{3} \mathrm{Si}_{42} \mathrm{Zr}_{55}$ & $\tau_{1}+\alpha\left(\mathrm{Si}_{4} \mathrm{Zr}_{5}\right)+\mathrm{Si}_{2} \mathrm{Zr}_{3}$ & 34.5 & 32.5 & 45.5 & 54.1 & 38.9 & 60.6 \\
\hline $\mathrm{Fe}_{27} \mathrm{Si}_{38} \mathrm{Zr}_{35}$ & $\tau_{9}+\alpha\left(\mathrm{Si}_{4} \mathrm{Zr}_{5}\right)+\tau_{1}$ & 40.7 & 30.5 & 45.7 & 54.0 & 34.8 & 33.8 \\
\hline $\mathrm{Fe}_{29.5} \mathrm{Si}_{23} \mathrm{Zr}_{47.5}$ & $\alpha\left(\mathrm{Fe}_{2} \mathrm{Zr}\right)+\mathrm{SiZr}_{2}+\mathrm{Si}_{2} \mathrm{Zr}_{3}$ & 8.7 & 32.9 & 33.6 & 64.4 & 38.8 & 60.8 \\
\hline $\mathrm{Fe}_{10} \mathrm{Si}_{10} \mathrm{Zr}_{80}$ & $\beta \mathrm{Zr}+\mathrm{SiZr}_{2}+$ liquid & 0 & 95.9 & 30.6 & 65.8 & 0.2 & 72.6 \\
\hline $\mathrm{Fe}_{62.5} \mathrm{Si}_{16.5} \mathrm{Zr}_{21}$ & $\tau_{5}+\alpha\left(\mathrm{Fe}_{2} \mathrm{Zr}\right)$ & 17.5 & 20.5 & 13.2 & 25.2 & $\ldots$ & $\ldots$ \\
\hline $\mathrm{Fe}_{33} \mathrm{Si}_{42} \mathrm{Zr}_{25}$ & $\tau_{4}+\tau_{9}$ & 40.7 & 18.2 & 42.4 & 27.9 & $\ldots$ & $\ldots$ \\
\hline $\mathrm{Fe}_{39} \mathrm{Si}_{48} \mathrm{Zr}_{13}$ & $\varepsilon(\mathrm{FeSi})+\tau_{9}$ & 50.2 & 0.1 & 43.1 & 27.8 & $\ldots$ & $\ldots$ \\
\hline $\mathrm{Fe}_{45} \mathrm{Si}_{25} \mathrm{Zr}_{30}$ & $\alpha\left(\mathrm{Fe}_{2} \mathrm{Zr}\right)+\tau_{1}$ & 15.5 & 27.1 & 34.2 & 30.9 & $\ldots$ & $\ldots$ \\
\hline $\mathrm{Fe}_{81.5} \mathrm{Si}_{2.5} \mathrm{Zr}_{17}$ & $\gamma \mathrm{Fe}+\alpha\left(\mathrm{Fe}_{2} \mathrm{Zr}\right)$ & 0.5 & 0.6 & 5.0 & 26.7 & $\cdots$ & $\ldots$ \\
\hline $\mathrm{Fe}_{62} \mathrm{Si}_{20} \mathrm{Zr}_{18}$ & $\alpha \mathrm{Fe}+\tau_{5}$ & 22.8 & 20.0 & 8.3 & 1.5 & $\ldots$ & $\ldots$ \\
\hline $\mathrm{Fe}_{70} \mathrm{Si}_{17} \mathrm{Zr}_{13}$ & $\alpha \mathrm{Fe}+\tau_{5}$ & 9.8 & 3.1 & 22.5 & 20.1 & $\ldots$ & $\ldots$ \\
\hline $\mathrm{Fe}_{43.5} \mathrm{Si}_{25} \mathrm{Zr}_{31.5}$ & $\tau_{1}+\tau_{2}$ & 32.7 & 33.2 & 17.4 & 30.8 & $\ldots$ & $\ldots$ \\
\hline $\mathrm{Fe}_{61.5} \mathrm{Si}_{11.5} \mathrm{Zr}_{27}$ & $\alpha\left(\mathrm{Fe}_{2} \mathrm{Zr}\right)$ & 10.6 & 29.1 & $\ldots$ & $\ldots$ & $\ldots$ & $\ldots$ \\
\hline
\end{tabular}

Seven analyses were carried out for each phase and the standard deviation of the measured concentration is \pm 0.5 at.\%

the dark gray and the black represent $\tau_{5}\left(\mathrm{Fe}_{16} \mathrm{Si}_{7} \mathrm{Zr}_{6}\right)$ phase, $\alpha\left(\mathrm{Fe}_{2} \mathrm{Zr}\right)$ phase and $\gamma \mathrm{Fe}$ phase, respectively. A two-phase equilibrium $\left(\tau_{4}\left(\mathrm{Fe}_{2} \mathrm{Si}_{2} \mathrm{Zr}\right)+\tau_{9}\right)$ was indentified in the $\mathrm{Fe}_{35} \mathrm{Si}_{40} \mathrm{Zr}_{25}$ (at.\%) alloy at $1273 \mathrm{~K}$ for $1440 \mathrm{~h}$, and indicated in Fig. 3(b), where the $\tau_{9}$ phase with the strip shape distributes in the $\tau_{4}$ matrix. Figure 3(c) presents the two-phase microstructure $\left(\tau_{5}\left(\mathrm{Fe}_{16} \mathrm{Si}_{7} \mathrm{Zr}_{6}\right)+\alpha \mathrm{Fe}\right)$ of the $\mathrm{Fe}_{71} \mathrm{Si}_{15} \mathrm{Zr}_{14}$ (at.\%) alloy equilibrated at $1273 \mathrm{~K}$ for $1440 \mathrm{~h}$.

In the $\mathrm{Fe}_{20} \mathrm{Si}_{70} \mathrm{Zr}_{10}$ (at.\%) alloy annealed at $1373 \mathrm{~K}$ for $720 \mathrm{~h}$, the three-phase microstructure $\left(\xi_{\alpha}\left(\mathrm{FeSi}_{2}\right)+\mathrm{Si}+\right.$ $\mathrm{Si}_{2} \mathrm{Zr}$ ) was observed, as shown in Fig. 3(d). Figure 3(e) shows the three-phase microstructure $\left((\mathrm{Zr}, \mathrm{Fe})_{5} \mathrm{Si}_{3}+\right.$ $\left.\varepsilon(\mathrm{FeSi})+\tau_{4}\left(\mathrm{Fe}_{2} \mathrm{Si}_{2} \mathrm{Zr}\right)\right)$ of the $\mathrm{Fe}_{50} \mathrm{Si}_{40} \mathrm{Zr}_{10}$ (at.\%) alloy annealed at $1373 \mathrm{~K}$ for $720 \mathrm{~h}$. In the $\mathrm{Fe}_{3} \mathrm{Si}_{42} \mathrm{Zr}_{55}$ (at.\%) alloy quenched from $1373 \mathrm{~K}$ for $720 \mathrm{~h}$, the three-phase microstructure $\left(\mathrm{Si}_{2} \mathrm{Zr}_{3}+\tau_{1} \quad(\mathrm{FeSiZr})+\alpha \quad\left(\mathrm{Si}_{4} \mathrm{Zr}_{5}\right)\right)$ was observed (Fig. 3f) and substantiated by the XRD result, as shown in Fig. 5(a), where the characteristic peaks of the
$\mathrm{Si}_{2} \mathrm{Zr}_{3}, \tau_{1}(\mathrm{FeSiZr})$ and $\alpha\left(\mathrm{Si}_{4} \mathrm{Zr}_{5}\right)$ phases were found and well marked by different symbols. In the $\mathrm{Fe}_{5} \mathrm{Si}_{55} \mathrm{Zr}_{40}$ (at.\%) alloy annealed at $1373 \mathrm{~K}$ for $720 \mathrm{~h}$, the three-phase

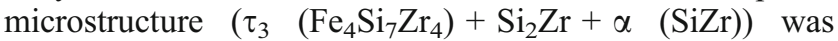
observed (Fig. 4a) and substantiated by XRD result, as shown in Fig. 5(b), where the characteristics peaks of $\tau_{3}\left(\mathrm{Fe}_{4} \mathrm{Si}_{7} \mathrm{Zr}_{4}\right), \mathrm{Si}_{2} \mathrm{Zr}$ and $\alpha(\mathrm{SiZr})$ are confirmed and well marked by different symbols. In the $\mathrm{Fe}_{33.5} \mathrm{Si}_{43.5} \mathrm{Zr}_{23}$ (at.\%) alloy annealed at $1373 \mathrm{~K}$ for $720 \mathrm{~h}$, the three-phase equilibrium of $\left(\tau_{4}\left(\mathrm{Fe}_{2} \mathrm{Si}_{2} \mathrm{Zr}\right)+\tau_{9}+\varepsilon(\mathrm{FeSi})\right)$ was found, as presented in Fig. 4(b). In addition, the three-phase equilibrium of $\left((\mathrm{Zr}, \mathrm{Fe})_{5} \mathrm{Si}_{3}+\beta\left(\mathrm{Fe}_{2} \mathrm{Si}\right)+\varepsilon(\mathrm{FeSi})\right)$ was identified in the $\mathrm{Fe}_{57} \mathrm{Si}_{40} \mathrm{Zr}_{3}$ (at.\%) alloy annealed at $1373 \mathrm{~K}$ for $720 \mathrm{~h}$, and its equilibrium microstructure is presented in the Fig. 4(c). As illustrated in Fig. 4(d), the three-phase equilibrium microstructures $\left(\tau_{3}\left(\mathrm{Fe}_{4} \mathrm{Si}_{7} \mathrm{Zr}_{4}\right)+\mathrm{Si}_{2} \mathrm{Zr}+\xi_{\alpha}\right.$ $\left(\mathrm{FeSi}_{2}\right)$ ) was detected in the $\mathrm{Fe}_{20} \mathrm{Si}_{60} \mathrm{Zr}_{20}$ (at.\%) alloys quenched from $1373 \mathrm{~K}$ for $720 \mathrm{~h}$. As shown in Fig. 4(e), a 
Table 3 Equilibrium compositions of the Fe-Si-Zr system determined at $1373 \mathrm{~K}$

\begin{tabular}{|c|c|c|c|c|c|c|c|}
\hline \multirow[b]{3}{*}{ Alloy, at.\% } & \multirow{3}{*}{$\begin{array}{c}\text { Phase equilibrium } \\
\text { Phase } 1+\text { Phase } 2+\text { Phase } 3\end{array}$} & \multicolumn{6}{|c|}{ Composition, at. $\%$} \\
\hline & & \multicolumn{2}{|c|}{ Phase 1} & \multicolumn{2}{|c|}{ Phase 2} & \multicolumn{2}{|c|}{ Phase 3} \\
\hline & & $\mathbf{S i}$ & $\mathbf{Z r}$ & $\mathbf{S i}$ & $\mathbf{Z r}$ & $\mathrm{Si}$ & $\mathbf{Z r}$ \\
\hline $\mathrm{Fe}_{20} \mathrm{Si}_{70} \mathrm{Zr}_{10}$ & $(\mathrm{Si})+\xi_{\alpha}\left(\mathrm{FeSi}_{2}\right)+\mathrm{Si}_{2} \mathrm{Zr}$ & 99.5 & 0.1 & 66.8 & 7.2 & 63.5 & 29.5 \\
\hline $\mathrm{Fe}_{35} \mathrm{Si}_{55} \mathrm{Zr}_{10}$ & $\xi_{\alpha}\left(\mathrm{FeSi}_{2}\right)+\varepsilon(\mathrm{FeSi})+\tau_{3}$ & 66.3 & 7.7 & 51.2 & 0.2 & 51.5 & 23.4 \\
\hline $\mathrm{Fe}_{20} \mathrm{Si}_{60} \mathrm{Zr}_{20}$ & $\xi_{\alpha}\left(\mathrm{FeSi}_{2}\right)+\mathrm{Si}_{2} \mathrm{Zr}+\tau_{3}$ & 65.6 & 7.4 & 63.1 & 29.3 & 51.4 & 23.6 \\
\hline $\mathrm{Fe}_{5} \mathrm{Si}_{55} \mathrm{Zr}_{40}$ & $\mathrm{Si}_{2} \mathrm{Zr}+\alpha(\mathrm{SiZr})+\tau_{3}$ & 63.8 & 29.7 & 50.5 & 49.2 & 52.1 & 24.3 \\
\hline $\mathrm{Fe}_{27.5} \mathrm{Si}_{49} \mathrm{Zr}_{23.5}$ & $\varepsilon(\mathrm{FeSi})+\tau_{3}+\tau_{9}$ & 50.5 & 0.2 & 50.9 & 24.1 & 43.5 & 27.8 \\
\hline $\mathrm{Fe}_{33.5} \mathrm{Si}_{43.5} \mathrm{Zr}_{23}$ & $\varepsilon(\mathrm{FeSi})+\tau_{4}+\tau_{9}$ & 49.9 & 0.6 & 40.3 & 19.5 & 43.4 & 27.7 \\
\hline $\mathrm{Fe}_{50} \mathrm{Si}_{40} \mathrm{Zr}_{10}$ & $\varepsilon(\mathrm{FeSi})+(\mathrm{Zr}, \mathrm{Fe})_{5} \mathrm{Si}_{3}+\tau_{4}$ & 50.5 & 0 & 36.4 & 11.3 & 41.0 & 18.8 \\
\hline $\mathrm{Fe}_{57} \mathrm{Si}_{40} \mathrm{Zr}_{3}$ & $\varepsilon(\mathrm{FeSi})+(\mathrm{Zr}, \mathrm{Fe})_{5} \mathrm{Si}_{3}+\beta\left(\mathrm{Fe}_{2} \mathrm{Si}\right)$ & 49.7 & 0 & 36.3 & 10.6 & 34.6 & 0 \\
\hline $\mathrm{Fe}_{43} \mathrm{Si}_{38} \mathrm{Zr}_{19}$ & $\tau_{4}+(\mathrm{ZrFe})_{5} \mathrm{Si}_{3}+\tau_{9}$ & 40.9 & 19.2 & 36.5 & 11.2 & 42.3 & 28.8 \\
\hline $\mathrm{Fe}_{60} \mathrm{Si}_{30} \mathrm{Zr}_{10}$ & $\alpha \mathrm{Fe}+(\mathrm{Zr}, \mathrm{Fe})_{5} \mathrm{Si}_{3}+\tau_{8}$ & 27.0 & 0.1 & 36.2 & 11.3 & 29.9 & 13.8 \\
\hline $\mathrm{Fe}_{40} \mathrm{Si}_{35} \mathrm{Zr}_{25}$ & $\tau_{1}+\tau_{9}+\tau_{8}$ & 35.5 & 31.2 & 40.9 & 28.7 & 29.6 & 13.8 \\
\hline $\mathrm{Fe}_{7} \mathrm{Si}_{46} \mathrm{Zr}_{47}$ & $\alpha(\mathrm{SiZr})+\alpha\left(\mathrm{Si}_{4} \mathrm{Zr}_{5}\right)+\tau_{9}$ & 50.2 & 49.4 & 44.9 & 55.1 & 43.3 & 29.7 \\
\hline $\mathrm{Fe}_{68} \mathrm{Si}_{19} \mathrm{Zr}_{13}$ & $\alpha \mathrm{Fe}+\tau_{5}+\tau_{8}$ & 14.6 & 0 & 24.7 & 19.6 & 29.3 & 13.7 \\
\hline $\mathrm{Fe}_{51.5} \mathrm{Si}_{27.5} \mathrm{Zr}_{21}$ & $\tau_{1}+\tau_{5}+\tau_{8}$ & 34.1 & 33.0 & 25.1 & 20.2 & 28.7 & 14.4 \\
\hline $\mathrm{Fe}_{9} \mathrm{Si}_{36} \mathrm{Zr}_{55}$ & $\tau_{1}+\mathrm{Si}_{2} \mathrm{Zr}_{3}+\tau_{2}$ & 33.4 & 33.7 & 39.6 & 60.3 & 13.9 & 34.5 \\
\hline $\mathrm{Fe}_{68} \mathrm{Si}_{12} \mathrm{Zr}_{20}$ & $\gamma \mathrm{Fe}+\alpha\left(\mathrm{Fe}_{2} \mathrm{Zr}\right)+\tau_{5}$ & 2.9 & 0.2 & 12.5 & 24.7 & 13.7 & 20.3 \\
\hline $\mathrm{Fe}_{20} \mathrm{Si}_{20} \mathrm{Zr}_{60}$ & Liquid $+\mathrm{SiZr}_{2}+\alpha\left(\mathrm{Fe}_{2} \mathrm{Zr}\right)$ & 0.7 & 62.8 & 29.1 & 63.6 & 2.9 & 31.0 \\
\hline $\mathrm{Fe}_{2} \mathrm{Si}_{20} \mathrm{Zr}_{78}$ & $\beta \mathrm{Zr}+\mathrm{SiZr}_{2}+\mathrm{SiZr}_{3}$ & 0.1 & 97.3 & 29.8 & 63.8 & 24.8 & 74.1 \\
\hline $\mathrm{Fe}_{50} \mathrm{Si}_{35} \mathrm{Zr}_{15}$ & $\tau_{9}+(\mathrm{Zr}, \mathrm{Fe})_{5} \mathrm{Si}_{3}+\tau_{8}$ & 39.7 & 28.9 & 36.4 & 11.5 & 30.5 & 13.8 \\
\hline $\mathrm{Fe}_{19} \mathrm{Si}_{47} \mathrm{Zr}_{34}$ & $\tau_{3}+\alpha(\mathrm{SiZr})+\tau_{9}$ & 49.0 & 25.2 & 50.9 & 47.9 & 42.5 & 29.9 \\
\hline $\mathrm{Fe}_{3} \mathrm{Si}_{42} \mathrm{Zr}_{55}$ & $\tau_{1}+\alpha\left(\mathrm{Si}_{4} \mathrm{Zr}_{5}\right)+\mathrm{Si}_{2} \mathrm{Zr}_{3}$ & 33.9 & 34.2 & 44.4 & 55.4 & 39.6 & 60.3 \\
\hline $\mathrm{Fe}_{27} \mathrm{Si}_{38} \mathrm{Zr}_{35}$ & $\tau_{9}+\alpha\left(\mathrm{Si}_{4} \mathrm{Zr}_{5}\right)+\tau_{1}$ & 41.5 & 30.1 & 44.8 & 55.0 & 34.1 & 32.9 \\
\hline $\mathrm{Fe}_{29.5} \mathrm{Si}_{23} \mathrm{Zr}_{47.5}$ & $\alpha\left(\mathrm{Fe}_{2} \mathrm{Zr}\right)+\mathrm{SiZr}_{2}+\mathrm{Si}_{2} \mathrm{Zr}_{3}$ & 8.5 & 32.7 & 33.7 & 64.0 & 38.8 & 60.3 \\
\hline $\mathrm{Fe}_{49} \mathrm{Si}_{25} \mathrm{Zr}_{26}$ & $\tau_{1}+\tau_{5}+\alpha\left(\mathrm{Fe}_{2} \mathrm{Zr}\right)$ & 33.0 & 32.4 & 20.8 & 20.3 & 16.2 & 26.2 \\
\hline $\mathrm{Fe}_{10} \mathrm{Si}_{10} \mathrm{Zr}_{80}$ & $\beta Z r+\operatorname{SiZr}_{2}+$ liquid & 0 & 96.5 & 29.2 & 65.1 & 0.5 & 74.6 \\
\hline $\mathrm{Fe}_{53} \mathrm{Si}_{36} \mathrm{Zr}_{11}$ & $\mathrm{FeSi}+(\mathrm{Zr}, \mathrm{Fe})_{5} \mathrm{Si}_{3}+\beta\left(\mathrm{Fe}_{2} \mathrm{Si}\right)$ & 49.4 & 0 & 35.6 & 11.5 & 33.1 & 0 \\
\hline $\mathrm{Fe}_{34} \mathrm{Si}_{46} \mathrm{Zr}_{20}$ & $\varepsilon(\mathrm{FeSi})+\tau_{9}$ & 50.9 & 0.2 & 43.5 & 27.5 & $\ldots$ & $\ldots$ \\
\hline $\mathrm{Fe}_{62} \mathrm{Si}_{20} \mathrm{Zr}_{18}$ & $\alpha \mathrm{Fe}+\tau_{5}$ & 9.1 & 1.3 & 22.5 & 20.1 & $\ldots$ & $\ldots$ \\
\hline $\mathrm{Fe}_{45} \mathrm{Si}_{25} \mathrm{Zr}_{30}$ & $\alpha\left(\mathrm{Fe}_{2} \mathrm{Zr}\right)+\tau_{1}$ & 15.9 & 28.2 & 33.0 & 32.6 & $\ldots$ & $\ldots$ \\
\hline $\mathrm{Fe}_{62.5} \mathrm{Si}_{16.5} \mathrm{Zr}_{21}$ & $\tau_{5}+\alpha\left(\mathrm{Fe}_{2} \mathrm{Zr}\right)$ & 18.5 & 20.3 & 14.3 & 24.1 & $\cdots$ & $\ldots$ \\
\hline $\mathrm{Fe}_{81.5} \mathrm{Si}_{2.5} \mathrm{Zr}_{17}$ & $\gamma \mathrm{Fe}+\alpha\left(\mathrm{Fe}_{2} \mathrm{Zr}\right)$ & 0.4 & 0.6 & 4.1 & 26.6 & $\ldots$ & $\ldots$ \\
\hline $\mathrm{Fe}_{43} \mathrm{Si}_{25} \mathrm{Zr}_{32}$ & $\tau_{1}+\tau_{2}$ & 32.4 & 33.3 & 14.5 & 30.6 & $\ldots$ & $\ldots$ \\
\hline $\mathrm{Fe}_{67} \mathrm{Si}_{18} \mathrm{Zr}_{15}$ & $\alpha \mathrm{Fe}+\tau_{5}$ & 9.9 & 0.6 & 23.0 & 19.8 & $\ldots$ & $\ldots$ \\
\hline $\mathrm{Fe}_{71} \mathrm{Si}_{15} \mathrm{Zr}_{14}$ & $\alpha \mathrm{Fe}+\tau_{5}$ & 11.7 & 0.1 & 23.5 & 20.0 & $\ldots$ & $\ldots$ \\
\hline $\mathrm{Fe}_{60.5} \mathrm{Si}_{11.5} \mathrm{Zr}_{28}$ & $\alpha\left(\mathrm{Fe}_{2} \mathrm{Zr}\right)$ & 10.2 & 28.4 & $\ldots$ & $\ldots$ & $\ldots$ & $\ldots$ \\
\hline
\end{tabular}

Seven analyses were carried out for each phase and the standard deviation of the measured concentration is \pm 0.5 at.\%

two-phase equilibrium $\left(\varepsilon(\mathrm{FeSi})+\tau_{9}\right)$ was indentified in the $\mathrm{Fe}_{34} \mathrm{Si}_{46} \mathrm{Zr}_{20}\left(\right.$ at.\%) alloy at $1373 \mathrm{~K}$ for $720 \mathrm{~h}$, where the $\tau_{9}$ phase irregularly distributes in the $\varepsilon(\mathrm{FeSi})$ matrix, and the XRD result is indicated in Fig. 5(c). In the $\mathrm{Fe}_{19} \mathrm{Si}_{47} \mathrm{Zr}_{34}$ (at.\%) alloy quenched from $1373 \mathrm{~K}$ for $720 \mathrm{~h}$, the threephase microstructure $\left(\alpha(\mathrm{SiZr})+\tau_{9}+\tau_{3}\left(\mathrm{Fe}_{4} \mathrm{Si}_{7} \mathrm{Zr}_{4}\right)\right)$ was observed (Fig. 4f) and the XRD result was presented in Fig. 5(d). Therefore, the phase regions around the phase $\tau_{9}$ were measured by EPMA and known phases were identified by the component position. At this moment, it might infer the existence of a new phase. The effect of slightly differing compositions can be ignored, because the ternary-phase regions around the phase $\tau_{9}$ were large enough and the standard deviation of the measured concentration is \pm 0.5 at. $\%$. Through composition contrast, the new phase $\tau_{9}$ is not same as $\tau_{7}\left(\mathrm{Fe}_{2} \mathrm{Si}_{3} \mathrm{Zr}_{3}\right)$ given by Raghavan. ${ }^{[11]}$ Furthermore, In Fig. 5(c), the $\varepsilon(\mathrm{FeSi})$ phase is figured out by a circle symbol, and the rest peaks probably indicate the new phase $\tau_{9}$. If the characteristic peaks of $\alpha(\mathrm{SiZr})$ and $\tau_{3}\left(\mathrm{Fe}_{4} \mathrm{Si}_{7} \mathrm{Zr}_{4}\right)$ were removed in Fig. $5(\mathrm{~d})$, the rest peaks of them are exactly identical with the rests in Fig. 5(c). Thus, according to the EPMA and XRD results, the existence of phase $\tau_{9}$ at the temperature of $1373 \mathrm{~K}$ was confirmed in the Fe-Si-Zr ternary system. The crystal parameters of phase $\tau_{9}$ are under determination and will be revealed in our further report. 

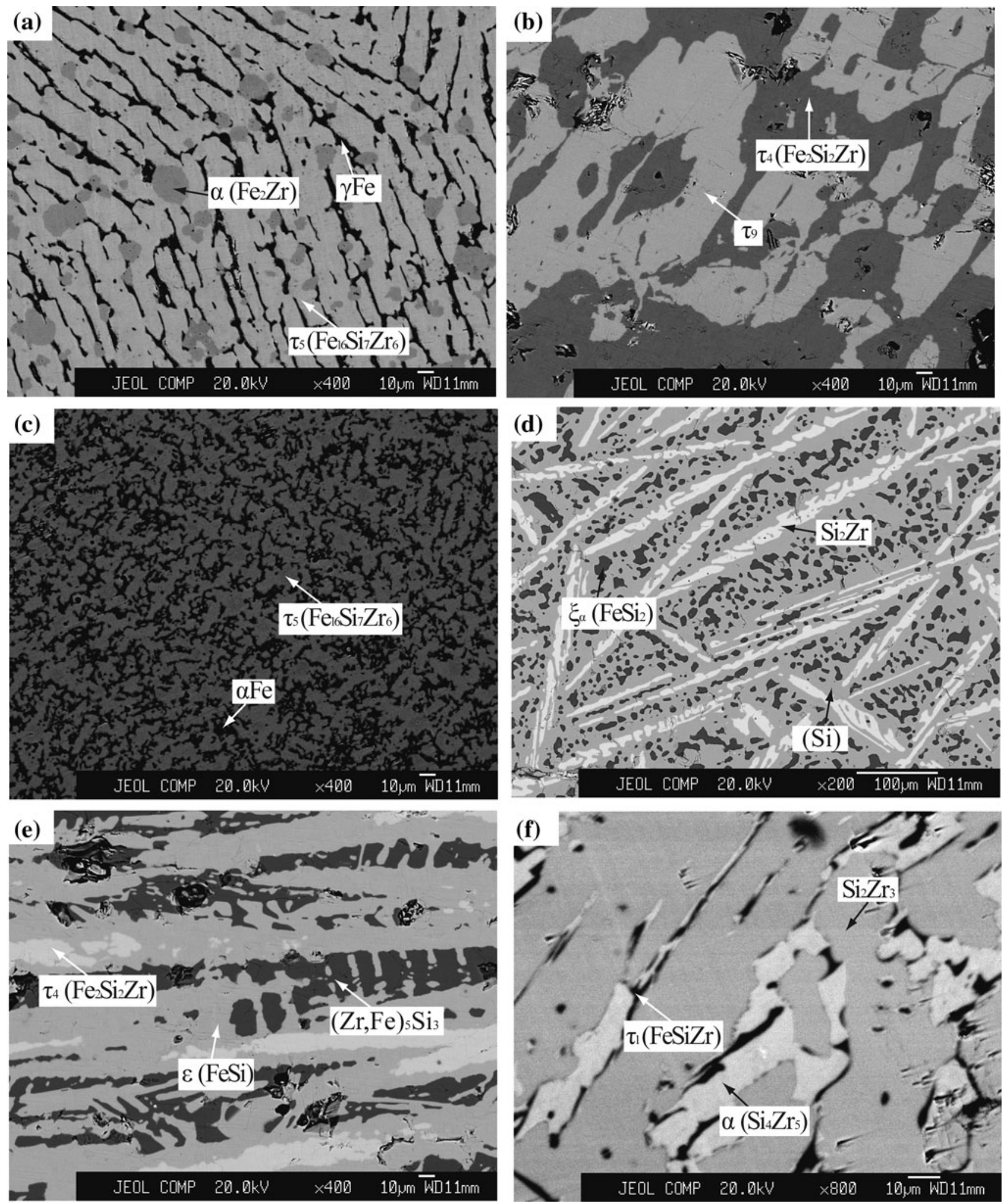

Fig. 3 BSE images of typical ternary alloys at $1273 \mathrm{~K}$ and $1373 \mathrm{~K}$ : (a) the $\mathrm{Fe}_{68} \mathrm{Si}_{12} \mathrm{Zr}_{20}$ (at.\%) alloy annealed at $1273 \mathrm{~K}$ for $1440 \mathrm{~h}$; (b) the $\mathrm{Fe}_{35} \mathrm{Si}_{40} \mathrm{Zr}_{25}$ (at.\%) alloy annealed at $1273 \mathrm{~K}$ for $1440 \mathrm{~h}$; (c) the $\mathrm{Fe}_{71} \mathrm{Si}_{15} \mathrm{Zr}_{14}$ (at.\%) alloy annealed at $1273 \mathrm{~K}$ for $1440 \mathrm{~h}$; (d) the $\mathrm{Fe}_{20} \mathrm{Si}_{70} \mathrm{Zr}_{10}$ (at.\%) alloy annealed at $1373 \mathrm{~K}$ for $720 \mathrm{~h}$; (e) the $\mathrm{Fe}_{50} \mathrm{Si}_{40} \mathrm{Zr}_{10}$ (at.\%) alloy annealed at $1373 \mathrm{~K}$ for $720 \mathrm{~h}$; and (f) the $\mathrm{Fe} \mathrm{S}_{3} \mathrm{~S}$ $\mathrm{i}_{42} \mathrm{Zr}_{55}$ (at.\%) alloy annealed at $1373 \mathrm{~K}$ for $720 \mathrm{~h}$ 

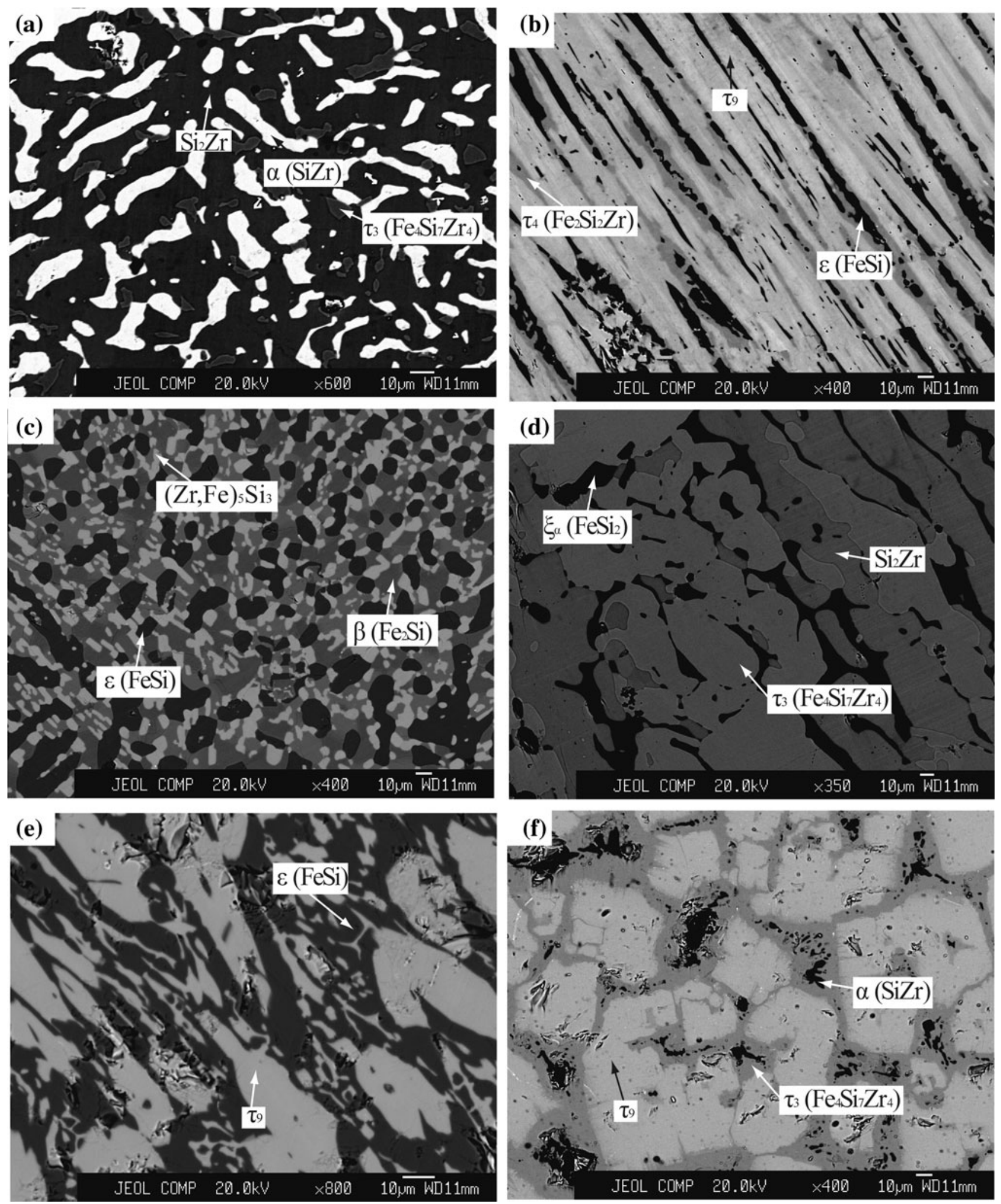

Fig. 4 BSE images of typical ternary alloys annealed at $1373 \mathrm{~K}$ for $720 \mathrm{~h}$ : (a) the $\mathrm{Fe}_{5} \mathrm{Si}_{55} \mathrm{Zr}_{40}$ (at.\%); (b) the $\mathrm{Fe}_{33.5} \mathrm{Si}_{43.5} \mathrm{Zr}_{23}$ (at.\%); (c) the $\mathrm{Fe}_{57} \mathrm{Si}_{40} \mathrm{Zr}_{3}$ (at.\%); (d) the $\mathrm{Fe}_{20} \mathrm{Si}_{60} \mathrm{Zr}_{20}$ (at.\%); (e) the $\mathrm{Fe}_{34} \mathrm{Si}_{46} \mathrm{Zr}_{20}$ (at.\%); and (f) the $\mathrm{Fe}_{19} \mathrm{Si}_{47} \mathrm{Zr}_{34}$ (at.\%) 

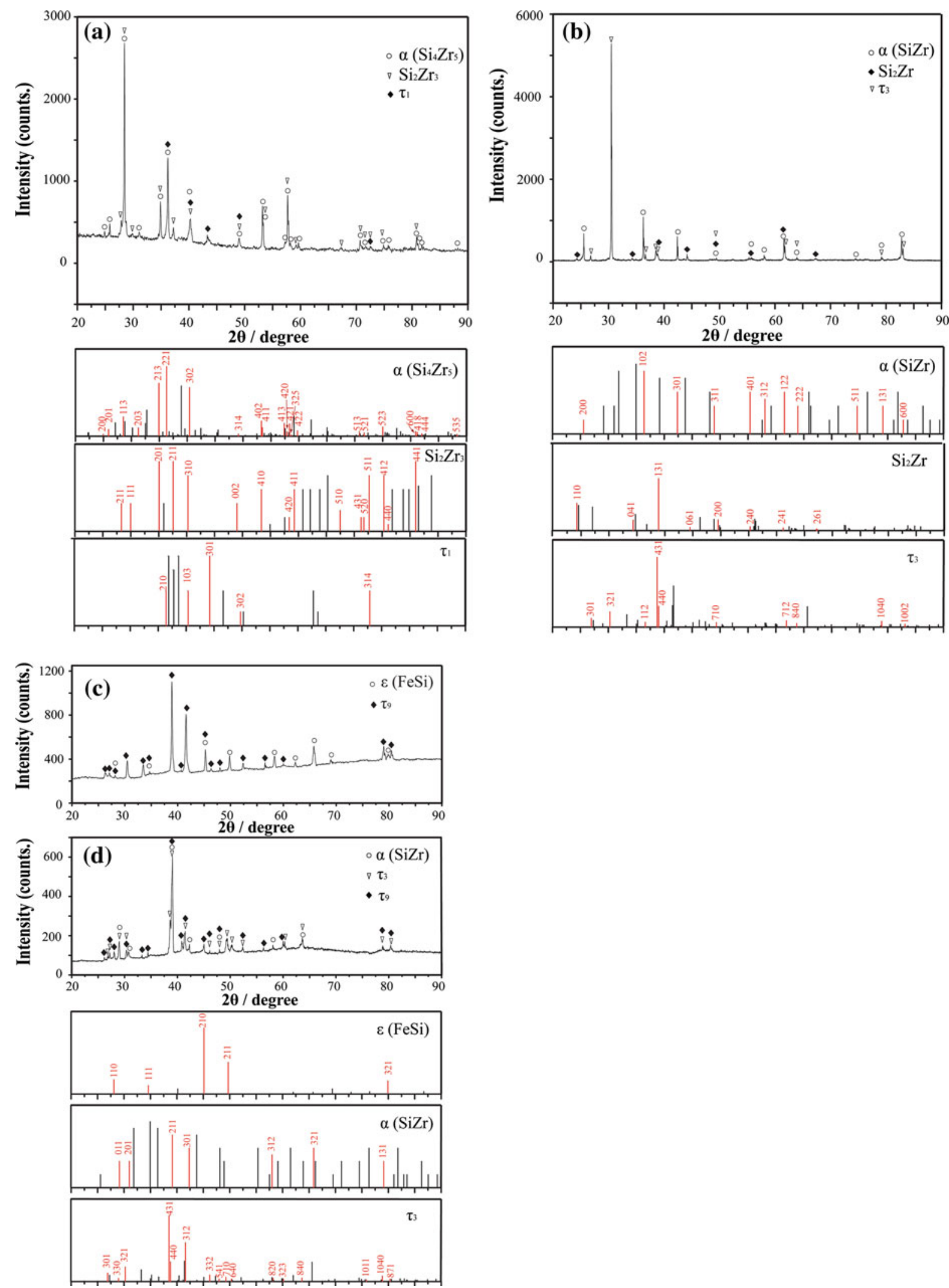

Fig. 5 XRD patterns obtained from alloys annealed at $1373 \mathrm{~K}$ for $720 \mathrm{~h}$ : (a) the $\mathrm{Fe}_{3} \mathrm{Si}_{42} \mathrm{Zr}_{55}$ (at.\%) alloy; (b) the $\mathrm{Fe}_{5} \mathrm{Si}_{55} \mathrm{Zr}_{40}$ (at.\%) alloy; (c) the $\mathrm{Fe}_{34} \mathrm{Si}_{46} \mathrm{Zr}_{20}$ (at.\%) alloy; and (d) the $\mathrm{Fe}_{19} \mathrm{Si}_{47} \mathrm{Zr}_{34}$ (at.\%) alloy. Vertical red marks with numbers indicate the position of possible Bragg reflections of the $\alpha\left(\mathrm{Si}_{4} \mathrm{Zr}_{5}\right), \mathrm{Si}_{2} \mathrm{Zr}_{3}, \tau_{1}, \alpha(\mathrm{SiZr}), \mathrm{Si}_{2} \mathrm{Zr}, \varepsilon(\mathrm{FeSi})$, and $\tau_{3}$ phase 

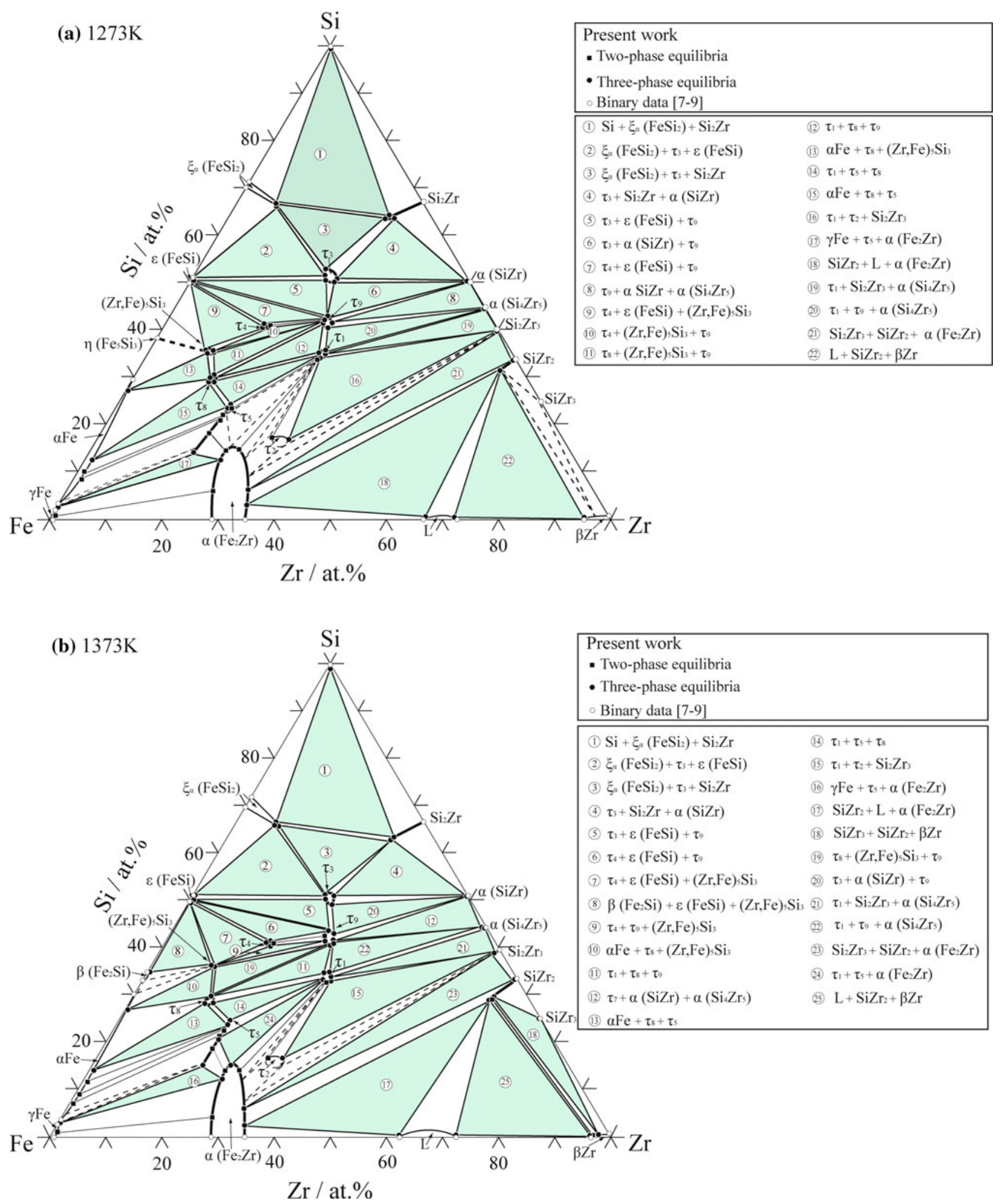

Fig. 6 Experimentally determined isothermal sections of the Fe-Si-Zr system at: (a) $1273 \mathrm{~K}$ and (b) $1373 \mathrm{~K}$ 


\subsection{Isothermal Section}

All the equilibrium compositions of the Fe-Si-Zr ternary system at 1273 and $1373 \mathrm{~K}$ determined by EPMA were listed in Tables 2 and 3, respectively. Based on the experimental data mentioned above, two isothermal sections at 1273 and $1373 \mathrm{~K}$ were constructed in Fig. 6(a) and (b), respectively.

In the isothermal section at $1273 \mathrm{~K}$ shown in Fig. 6(a), several ternary phases were experimentally determined and a new phase $\tau_{9}$ was observed for the first time in this work. The solubility of $\mathrm{Zr}$ in the $\xi_{\alpha}\left(\mathrm{FeSi}_{2}\right)$ phase is found to be 7.5 at.\%, the solubility of $\mathrm{Fe}$ in the $\mathrm{Si}_{2} \mathrm{Zr}$ is measured to be 8 at.\% and the solubility of $\mathrm{Fe}$ in the $\mathrm{SiZr}_{2}$ phases is shown to be 4 at.\%. Furthermore, the solubility of $\mathrm{Si}$ in the $\mathrm{Fe}_{2} \mathrm{Zr}$ phase is found to be about 16 at.\%. Compared with isothermal section at $1073 \mathrm{~K}$ shown in Fig. 1, the single-phase regions of the ternary phases $\left(\tau_{1}, \tau_{3}, \tau_{4}, \tau_{8}\right)$ identified in the isothermal section at $1273 \mathrm{~K}$, change to be one-phase regions. On the other hand, the $\tau_{5}$ phase changes to be a linear compound, and the $\mathrm{Zr}$ content in the $\tau_{5}$ phase is 20 at.\%. In addition, the ternary phase named $\tau_{9}$ indicated in Fig. 2(b), (d) and (e), separates the ternary region $\left(\varepsilon(\mathrm{FeSi})+\tau_{3}+\tau_{4}\right)$ into two new ternary regions: $\left(\varepsilon(\mathrm{FeSi})+\tau_{3}+\tau_{9}\right)$ and $\left(\varepsilon(\mathrm{FeSi})+\tau_{4}+\tau_{9}\right)$. Compared with isothermal section given by Raghavan, ${ }^{[11]}$ the phase $(\mathrm{Fe}, \mathrm{Si})_{2} \mathrm{Zr}, \tau_{6}\left(\mathrm{Fe}_{5} \mathrm{Si}_{12} \mathrm{Zr}_{3}\right)$ and $\tau_{7}\left(\mathrm{Fe}_{2} \mathrm{Si}_{3} \mathrm{Zr}_{3}\right)$ disappear, as well as a continuous series of solid solution formed by $\mathrm{FeZr}_{2}$ and $\mathrm{SiZr}_{2}$. Also, the solubility of $\mathrm{Si}$ in the $\mathrm{Fe}_{2} \mathrm{Zr}$ phase and the solubility of $\mathrm{Zr}$ in the $\xi_{\alpha}\left(\mathrm{FeSi}_{2}\right)$ phase increase. For the phase $\tau_{9}$, although the standard deviation of the measured concentration is taken into consideration, the composition position of $\tau_{9}$ is still far away from known ternary phase $\left(\tau_{1}(\mathrm{FeSiZr}), \tau_{3}\left(\mathrm{Fe}_{4} \mathrm{Si}_{4} \mathrm{Zr}_{7}\right), \tau_{7}\left(\mathrm{Fe}_{2} \mathrm{Si}_{3} \mathrm{Zr}_{3}\right)\right)$. Moreover, through XRD patterns, the unknown peaks cannot be calibrated by other peaks of $\mathrm{Fe}-\mathrm{Si}-\mathrm{Zr}$ metal compounds. Therefore, it is indicated that the phase $\tau_{9}$ exists.

The phase relationships in the isothermal section at $1373 \mathrm{~K}$ (Fig. 6b) are similar to that at $1273 \mathrm{~K}$. Compared with the isothermal section at $1273 \mathrm{~K}$, the apparent difference happens in the binary $\mathrm{Fe}-\mathrm{Zr}$ side, where $\mathrm{Si}$ content varies from 28 to 50 at.\%. The $\eta\left(\mathrm{Fe}_{5} \mathrm{Si}_{3}\right)$ phase disappears and the $\beta\left(\mathrm{Fe}_{2} \mathrm{Si}\right)$ appears, which leads to appearance of a ternary region $\left((\mathrm{Zr}, \mathrm{Fe})_{5} \mathrm{Si}_{3}+\varepsilon(\mathrm{FeSi})+\beta\right.$ $\left.\left(\mathrm{Fe}_{2} \mathrm{Si}\right)\right)$. It can be seen from the $\mathrm{Fe}-\mathrm{Si}$ binary phase diagram that, the $\mathrm{Fe}_{5} \mathrm{Si}_{3}$ only exists in the temperature range between $1098 \mathrm{~K}$ and $1333 \mathrm{~K}$. According to the previous and current study, $\mathrm{Zr}$ can stabilize a ternary phase of the $\mathrm{Fe}_{5} \mathrm{Si}_{3}$ structure ${ }^{[10]}$ and make presence of $\mathrm{Fe}_{5} \mathrm{Si}_{3}$ to be extended within larger temperature range. The solubility of $\mathrm{Fe}$ in the $\mathrm{SiZr}_{2}$ phases increases, being up to 8 at. $\%$ at $1373 \mathrm{~K}$. Additionally, the liquid region in the $\mathrm{Zr}$-rich corner becomes larger.

\section{Conclusions}

The phase equilibria of the Fe-Si-Zr alloy system at 1273 and $1373 \mathrm{~K}$ were experimentally determined in the whole compositional range, which consists of a series of ternary phase equilibria, two phase equilibria and solid solutions. The newly determined phase equilibria will provide additional support for the thermodynamic assessment of this Fe-Si-Zr system and compositional design of new silicon steels. The important conclusions drawn from the present study are as follows:

(1) The previously-reported phases $\tau_{1}, \tau_{2}, \tau_{3}, \tau_{4}, \tau_{5}, \tau_{8}$ and $(\mathrm{Zr}, \mathrm{Fe})_{5} \mathrm{Si}_{3}$ were found both at $1273 \mathrm{~K}$ and $1373 \mathrm{~K}$. The phases $\tau_{6}$ and $\tau_{7}$ were not found at either temperature. Also, $\mathrm{FeZr}_{2}$ and $\mathrm{SiZr}_{2}$ do not form a continuous solid solution at 1273 and $1373 \mathrm{~K}$. A new phase denoted here as $\tau_{9}$ was found at both temperatures.

(2) The measured homogeneity range of the newly found phase $\tau_{9}(29 \mathrm{Fe} 42 \mathrm{Si} 29 \mathrm{Zr})$ is sufficiently narrow to conclude that it is distinctly different from the ternary phase $\tau_{7}$ denoted as $\tau_{6}$ by Raghavan ${ }^{[11]}$ with the nominal composition $\mathrm{Fe}_{2} \mathrm{Si}_{3} \mathrm{Zr}_{3}(25 \mathrm{Fe} 37.5 \mathrm{Si} 37.5 \mathrm{Zr})$. Available structural evidence supports this conclusion.

(3) The measured composition of the $\tau_{8}$ phase $(57 \mathrm{Fe} 29 \mathrm{~S}-$ i14Zr) agrees with the formula $\mathrm{Fe}_{4} \mathrm{Si}_{2} \mathrm{Zr}$ found in the structural investigations of Yarmolyuk et al ${ }^{[20,21]}$ and listed in the review of Raghavan. ${ }^{[11]}$

\section{Acknowledgments}

Authors wish to acknowledge the National Natural Science Foundation of China (Grant No. 51031003, Grant No. 51171159), the Ministry of Education, PR CHINA (Grant No. 20120121130004), National Key Basic Research Program of China (973 Program, 2012CB825700) and Aviation Industry Corporation of China for funding this research.

\section{References}

1. C.M.B. Bacaltchuk, G.A. Castello-Branco, M. Ebrahimi, H. Garmestani, and A.D. Rollett, Effect of Magnetic Field Applied During Secondary Annealing on Texture and Grain Size of Silicon Steel, Scr. Mater, 2003, 48, p 1343-1347

2. N. Takahashi and J. Harase, Recent Development of Technology of Grain Oriented Silicon Steel, Mater. Sci. Forum., 1996, 204-206, p 143-154

3. C.W. Chen, Magnetism and Metallurgy of Soft Magnetic Materials, Dove Publications, New York, 1986, p 377

4. F.G. Caballero, M.K. Miller, S.S. Babu, and C. Garcia-Mateo, Atom Scale Observations of Bainite Transformation in a high Carbon high Silicon Steel, Acta Mater., 2007, 55, p 381-390

5. K. Matsumura and B. Fukuda, Recent Developments of Nonoriented Electrical Steel Sheets, IEEE Trans. Magn., 1984, 20, p 1533-1538

6. Z.Z. He, Cold Rolled Non Grain Oriented Steel, Electrical Steel, Z.Z. He, Ed., Metallurgical-Industry Publishing, Beijing, 1997, p 416-417, in Chinese

7. O. Kubaschewski, Iron-Binary Phase Diagrams, Springer, New York, 1982, p 1-4

8. F. Stein, G. Sauthoff, and M. Palm, Experimental Determination of Intermetallic Phases, Phase Equilibria, and Invariant 
Reaction Temperatures in the Fe-Zr System, J. Phase Equilib., 2002, 23, p 480-494

9. H. Okamoto, The Si-Zr (Silicon-Zirconium) System, Bull. Alloy Phase Diagr., 1990, 11, p 513-519

10. L.A. Lisenko, Z. Ban, and E.I. Gladisevskii, Investigation of the System Zr-Fe-Si, Croat. Chem. Acta., 1971, 43, p 113-118

11. V. Raghavan, The Fe-Si-Zr (Iron-Silicon-Zirconium) System, Phase Diagrams of Ternary Iron Alloys, Part 6B, Indian Institute of Metals, Calcutta, 1992, p 1189-1194

12. Y. Du, W. Xiong, W. Zhang, H. Chen, and W. Sun, IronSilicon-Zirconium, Landolt-Bornstein New Series IV, Vol 11D5, 2009, p 605-620

13. W. Jeitschko, A.G. Jordan, and P.A. Beck, V and E Phases in Ternary Systems with Transition Metals and Silicon or Germanium, Trans. Met. Soc. AIME, 1969, 245, p 335-339

14. R.C. Mittal, S.K. Si, and K.P. Gupta, Si-Stabilised C14 Laves Phases in the Transaction Metal Systems, J. Less-Common Met., 1978, 60, p 75-82

15. Ch.B.H. Evers and W. Jeitschko, Crystal Structure of Tetrazirconium Tetrairon Heptasilicide, $\mathrm{Zr}_{4} \mathrm{Fe}_{4} \mathrm{Si}_{7}$, Z. Kristallogr., 1996, 211, p 119

16. Yu.V. Voroshilov, V.Ya. Markiv, and E.I. Gladyshevskii, The Zirconium-Nickel-Silicon System, Izv. Akad. Nauk SSSR, Neorg. Mater., 1967, 3(8), p 1140-1148, in Russian
17. P. Chaudouet, B. Lambert, R. Madar, and R. Fruchart, Existence of a G-type Phase in the Fe-Zr-Si System, Ann. Chi. Fr., 1984, 9(2), p 119-121

18. L.A. Lysenko, Z. Ban, Ya.P. Yarmolyuk, and E.I. Gladisevskii, Reaction of $\mathrm{Zr}$ with Fe-Group Transition Metals and Si, Struktura Faz. Faz. Prevrashcheniya i Diagrammy Sostoyaniya Metal. Sistem., O.S. Ivanov, Ed., Nauka, Moscow, 1974, p 21-25, in Russian

19. Y.P. Yarmolyuk, Y.N. Grin, and E.I. Gladyshevskii, Crystal Structure of $\mathrm{Hf}_{3} \mathrm{Ni}_{2} \mathrm{Si}_{3}$, Sov. Phys. Crystallogr., 1977, 22, p 416-419, in Russian

20. Y.P. Yarmolyuk, L.A. Lysenko, and E.I. Gladyshevskii, The $\mathrm{ZrFe}_{4} \mathrm{Si}_{2}$ Crystal Structure: A New Structure Type in the Ternary Silicides of Transition Metals, Dopov. Akad. Nauk Ukrain. RSA(A), 1975, p 279-282, in Ukrainian

21. Y.P. Yarmolyuk, L.A. Lysenko, and E.I. Gladyshevskii, Crystal Structure of New Ternary Compounds in the Zr-FeSi System, Tezisy Dokl.-Vses. Konf. Kristallokhim. Intermetall. Soedin., 2nd ed., R.M. Rykhal, Ed., L'Vov Gos. Univ., USSR, 1974, p 21-22

22. O.Z. Blazina and R. Trojko, On Friauf-Laves Phases in the $\mathrm{Zr}_{1-x} \mathrm{Al}_{x} \mathrm{~T}_{2}, \mathrm{Zr}_{1-x} \mathrm{Si}_{x} \mathrm{~T}_{2}$, and $\mathrm{Zr}_{1-x} \mathrm{Ti}_{x} \mathrm{~T}_{2} \quad(\mathrm{~T}=\mathrm{Mn}$, Fe, Co) Systems, J. Less-Common Met., 1987, 133, p $277-$ 286 